\title{
Ultrathin uniform platinum nanowires via a facile route using an inverse hexagonal surfactant phase template
}

Article

Accepted Version

Akbar, S., Boswell, J., Worsley, C., Elliott, J. M. and Squires, A. M. (2018) Ultrathin uniform platinum nanowires via a facile route using an inverse hexagonal surfactant phase template. Langmuir, 34 (24). pp. 6991-6996. ISSN 1520-5827 doi: https://doi.org/10.1021/acs.langmuir.7b03970 Available at https://centaur.reading.ac.uk/77867/

It is advisable to refer to the publisher's version if you intend to cite from the work. See Guidance on citing.

To link to this article DOI: http://dx.doi.org/10.1021/acs.langmuir.7b03970

Publisher: American Chemical Society

All outputs in CentAUR are protected by Intellectual Property Rights law, including copyright law. Copyright and IPR is retained by the creators or other copyright holders. Terms and conditions for use of this material are defined in the End User Agreement.

www.reading.ac.uk/centaur 
Central Archive at the University of Reading

Reading's research outputs online 


\section{Ultrathin uniform platinum nanowires via a facile}

\section{route using an inverse hexagonal surfactant phase}

\section{template}

Samina Akbar,${ }^{a, b}$ Jacob Boswell, ${ }^{c}$ Carys Worsley, ${ }^{c}$ Joanne M. Elliott, ${ }^{* a}$ and Adam M. Squires ${ }^{*} a c$

a Department of Chemistry, University of Reading, Whiteknights, Reading, RG6 6AD. Fax:

(+)44 (0)118 378 6331, E-mail: a.squires@bath.ac.uk, j.m.elliott@reading.ac.uk, Homepage:

http://www.reading.ac.uk/chemistry/chem-home.aspx

${ }^{\mathrm{b}}$ Department of Basic Sciences and Humanities, University of Engineering and Technology, KSK Campus, GT Road Lahore, Pakistan.

${ }^{c}$ Department of Chemistry, University of Bath, Bath BA2 7AY, UK

KEYWORDS. Self assembly, Inverse Hexagonal, Templating, Platinum, Nanowires

ABSTRACT. In this paper, we present an attractive method for the fabrication of long, straight, highly crystalline ultra-thin platinum nanowires. The fabrication is simply achieved using an inverse hexagonal $\left(H_{I I}\right)$ lyotropic liquid crystal phase of the commercial surfactant phytantriol as a template. A platinum precursor dissolved within the cylindrical aqueous channels of the liquid crystal phase is chemically reduced by galvanic displacement using stainless steel. We demonstrate the production of nanowires using the $H_{I I}$ phase in the phytantriol/water system which we obtain 
either by heating to $55^{\circ} \mathrm{C}$, or at room temperature by the addition of a hydrophobic liquid, 9-cistricosene, to relieve packing frustration. The two sets of conditions produced high aspect nanowires with diameters of $2.5 \mathrm{~nm}$ and $1.7 \mathrm{~nm}$ respectively, at least hundreds of nanometers in length, matching the size of the aqueous channels in which they grow. This versatile approach can be extended to produce highly uniform nanowires from a range of metals.

INTRODUCTION. Nanostructured platinum materials have been investigated for a wide range of high-surface-area applications. Some of these technologies are driven by the need to meet the increasing demand for renewable energy, for example, in fuel cells ${ }^{1}$ and solar cells ${ }^{2}$. Other applications include sensors ${ }^{3}$ and chemical catalysts ${ }^{4-5}$. The use of nanowires rather than the commonly used supported nanoparticles brings a number of advantages, overcoming several problems associated with the latter especially in fuel cells, such as support degradation, particle sintering, and limitations on catalyst loading ${ }^{1}$. Ultrathin platinum nanowires have been shown to exhibit higher electrochemical surface area activity than supported nanoparticles ${ }^{6}$, and greater electrochemical durability under voltage cycling ${ }^{5}$. In other applications, platinum nanowires bring new optical ${ }^{7}$, magnetic ${ }^{8}$ and catalytic properties ${ }^{9-10}$. More generally, ultrathin nanowires from other metals have applications in transparent conducting electrodes ${ }^{11}$.

A number of approaches have been used to produce ultrathin $(<10 \mathrm{~nm}$ diameter) platinum nanowires. Non-templating routes through chemical reduction generally give rise to shorter, more disordered wires ${ }^{6}$ although Xia et al. ${ }^{12}$ have reported the synthesis of $3 \mathrm{~nm}$ diameter ultralong nanowires in a template-free solvothermal route taking 1-2 days, and involving temperatures of $170{ }^{\circ} \mathrm{C}$. Longer, straight ultrathin platinum nanowires are more commonly produced by growing the metal within the cylindrical pores of a template such as mesoporous 
silica $^{13}$, carbon nanofibers ${ }^{14}$, insulin fibrils ${ }^{5}$ and metal organic frameworks ${ }^{15}$. However, the assembly and subsequent removal of these templates are associated with complex, multi-step processes, often involving harsh conditions and reagents.

Platinum nanomaterials with different morphologies showing periodicity in two or three dimensions have been produced by electrodeposition within highly ordered hexagonal or cubic lyotropic liquid crystalline phases ${ }^{16-18}$. They generally represent a much more attractive route than the templates mentioned in the previous paragraph. However, such lyotropic phases have not until now been used to produce straight ultra-thin nanowires (although inverse micelles have been used to grow nanowires which are much larger ${ }^{10}$ or more disordered ${ }^{19}$.

In this study, inverse hexagonal lyotropic liquid crystal phases $\left(H_{I I}\right)$ formed from self-assembly of the readily available lipid phytantriol (3,7,11,15-tetramethyl-1,2,3trihydroxy-hexadecane) are used for the template synthesis of ultra-thin, straight platinum nanowires. $H_{I I}$ phases contain arrays of straight aqueous channels a few nm in diameter, and have mainly been researched for pharmaceutical applications ${ }^{20}$. In the few cases where these have been used as templates, in the synthesis of metals ${ }^{21}$ and semiconductor ${ }^{22-23}$ nanowires, the nanowires produced are typically tens of nanometres in diameter; these dimensions are much larger than the diameter of the channels (indicating that the process has not faithfully 'templated' the channel dimension) and of the ultrathin nanowires we report in this paper. The $H_{I I}$ phase is the thermodynamically favoured phase for phytantriol in excess water above $45^{\circ} \mathrm{C}^{24}$. Addition of a hydrophobic liquid such as 9cis-tricosene (as here) or hexadecane ${ }^{25}$ relieves packing frustration by filling voids between the monolayer cylinders allowing the $H_{I I}$ phase to form at room temperature.

\section{EXPERIMENTAL.}


Materials and Equipment: 3,7,11,15-tetramethyl-1,2,3-hexadecanetriol $\left(\mathrm{C}_{20} \mathrm{H}_{42} \mathrm{O}_{3}\right)$, commonly known as phytantriol, was kindly donated by Adina Cosmetics Ingredients. Hexachloroplatinic acid (HCPA) solution ( $8 \mathrm{wt} \%$ in water) and 9-cis-tricosene were purchased from Aldrich. $0.5 \mathrm{M}$ sulfuric acid was prepared from Merck p.a.-grade concentrated acid. Laboratory reagent grade acetone was purchased from Fischer Scientific. $6 \mathrm{M} \mathrm{HCl}$ was prepared by diluting the laboratory reagent grade $\mathrm{HCl}(36 \%)$ from Fischer Scientific. All of the compounds were used as received. All solutions were prepared in purified water, which was prepared by passing deionized, distilled water through a Milli-Q water purification system. Stainless steel sheet ( $2 \mathrm{~mm}$ thick), purchased from Inspired steel limited, was cut down to $0.3-1 \mathrm{~cm}^{2}$ pieces by the in-house mechanical workshop.

Preparation of liquid crystal templates: The binary mixture of phytantriol containing $4.1 \%$ (w/w) 9-cis-tricosene was prepared by weighing appropriate amounts of phytantriol and 9-cistricosene in a capped glass vial. The mixture was vigorously stirred at room temperature with the help of a glass rod or polypropylene spatula. The glass vial containing the mixture was sealed and heated in water bath at $40{ }^{\circ} \mathrm{C}$ for $20 \mathrm{~min}$ and then cooled down to room temperature. The sample was stirred again. After mixing, the sample was allowed to stand for 1-2 hours to remove air bubbles. Phytantriol and the binary mixture of phytantriol/9-cis-tricosene were each dissolved in ethanol in 1:1 or 1:2 ratios by weight for SAXS analysis or platinum deposition on stainless steel respectively.

Fabrication of Pt-nanowires: For the fabrication of Pt-nanowires, a thick layer (1-2 mm) of phytantriol or binary mixture of phytantriol/9-cis-tricosene (dissolved in ethanol) was placed on the steel plate in a Teflon cell. The Teflon cell was left for 1-2 hour to allow the ethanol to evaporate. The Teflon cell was then sealed and placed inside a Pyrex water jacketed cell attached 
to a thermostat water bath for temperature control. After equilibration at the required temperature for 30-60 mins, HCPA solution was added drop wise to the top of the surfactant layer. It was allowed to stand for 4-8 hours until the mixture became fully black. Chemical deposition of Ptnanowires from $H_{I I}$ phase of phytantriol without the presence of tricosene was carried out at 55 ${ }^{\circ} \mathrm{C}$. In the presence of $4.1 \mathrm{wt} \%$ tricosene, deposition was carried out at $25^{\circ} \mathrm{C}$. Once the process was completed, the chemical deposits were removed from the substrate and transferred to a glass vial followed by washing (see below).

Washing of chemical deposits: Washing of chemical deposits was carried out with acetone (20 $\mathrm{ml})$, water $(10 \mathrm{ml}), 6 \mathrm{M} \mathrm{HCl}(10 \mathrm{ml})$, water $(10 \mathrm{ml})$, acetone $(10 \mathrm{ml})$ and finally with water. After washing, the nanostructured platinum powder was kept moist in order to avoid inhalation risks associated with dry powders.

Characterization:

Structural investigations were carried out by small angle X-ray scattering (SAXS) and transmission electron microscope (TEM) whereas electrochemical analysis was carried out by cyclic voltammetry.

Small Angle X-Ray Scattering (SAXS) analysis: Small angle X-ray scattering patterns were recorded using a Small Angle X-Ray System V 4.1.35 Bruker AXS operating at $45 \mathrm{kV}$ and 650 $\mu$ A. Silver behenate $\left[\mathrm{CH}_{3}\left(\mathrm{CH}_{2}\right)_{20} \mathrm{COOAg}\right]$ was used as a calibrant using $\mathrm{d} 001=5.8380 \mathrm{~nm}^{26}$. Scattering patterns were obtained with a wavelength of $0.154 \mathrm{~nm}$. During analysis the optics and the sample chamber were under vacuum to minimize the air scatter. 2-D scattering patterns were subsequently integrated and converted into one dimensional d-spacing intensity profiles by using Image J software with macros written in-house. The sample to detector distance was $107 \mathrm{~cm}$ (extended beam path) or $67 \mathrm{~cm}$ (short beam path). All the temperature controlled measurements 
were performed using an mri Physikalische Gerate GmbH high temperature heating stage (mri0065151/heating stage).

Sample preparation of nanostructured platinum materials: Small amounts of the Pt-nanowire powder were wrapped in polyimide sticky tape with the help of a spatula and attached to a metal frame for SAXS analysis.

Sample preparation of liquid crystals with incorporated platinum: During the chemical fabrication process, a small amount of a liquid crystal template along with the platinum replica was removed from the steel plate and a sandwich cell was prepared. A sample was sandwiched between two layers of a polyimide paper supported by a silicon rubber sheet attached together by double sided sticky tape. For temperature control, the cell was placed between two metal plates screwed together and then fixed into the heating stage.

Preparation of liquid crystal samples under excess hydration conditions: Thin coatings of phytantriol with or without the presence 9-cis-tricosene were deposited on the inside of open-ended thin-walled glass capillary tubes from their ethanolic solutions (w/w ratio of 1:1) followed by drying with compressed air $(\sim 15 \mathrm{~min})$ and then kept under ambient conditions for not less than an hour. Drying time with compressed air was extended to $\sim 30 \mathrm{~min}$ for immediate use (in this case, it was not kept for another hour under ambient conditions). During this time, the ethanol evaporated leaving a thin film $(21 \pm 5 \mu \mathrm{m}$ thick) of phytantriol with or without the presence of tricosene. The capillary was then filled with water or HCPA solution, sealed and fixed into the heating stage.

TEM Analysis: The surface morphology of the Pt-nanowires was revealed by taking images using Philips CM20 Analytical TEM and JEOL JEM-2010 HR TEM operated at $200 \mathrm{kV}$. For TEM analysis, $2 \mathrm{ml}$ of isopropyl ether or acetone was added to the sample in a glass vial and sonicated 
for 5 min to make a suspension of the Pt-nanowires. 1-2 drops of the suspension was placed on a copper grid with supporting carbon film (300 mesh) with the help of a dropper. The copper grid was placed on the sample holder and loaded inside the sample chamber for analysis.

Electrochemical characterisation: All electrochemical experiments were carried out on a purpose built electrochemical workstation and software, interfaced to a personal computer using a CIO-DAS08 data acquisition card (Measurement Computing), using data recording software written in-house. Electrochemical experiments were carried out in Pyrex water-jacketed cell of volume $\sim 5 \mathrm{ml}$ and sample temperature was controlled to $\pm 1{ }^{\circ} \mathrm{C}$ using thermostat water bath (Grant) which circulated water around the cell. All the glass ware used was cleaned using $1 \%$ solution of Micro-90 (Cole-Parmer) in which it was soaked for 24 hours followed by rinsing with distilled water. Final rinsing was carried out using deionized distilled water. All electrochemical experiments were carried out in a conventional three electrode system comprised of $\mathrm{Ag} / \mathrm{AgCl}$ reference electrode $(\mathrm{RE})$, platinum flag (area $\sim 1 \mathrm{~cm}^{2}$ ) counter electrode (CE) and a working electrode (WE). The platinum counter electrode was flamed until a colourless flame was observed.

The chemically deposited Pt-nanowire powder was attached abrasively to a bare gold wire electrode $(0.2 \mathrm{~mm}$ diameter). These electrodes were then used to record the cyclic voltammograms in $0.5 \mathrm{M} \mathrm{H}_{2} \mathrm{SO}_{4} \mathrm{vs} \mathrm{Ag} / \mathrm{AgCl}$ between the limits +1.2 to $-0.35 \mathrm{~V}$. The surface area of the rubbed Ptnanowire powder was measured by integrating the current in the hydride region during forward and backward scan, taking their average and divided by the conversion factor for platinum and scan rate. Before applying the platinum powder, the gold wire electrode was cycled in $0.5 \mathrm{H}_{2} \mathrm{SO}_{4}$ between the limits +1.7 to $-0.2 \mathrm{~V}$ vs. $\mathrm{Ag} / \mathrm{AgCl}$. All these experiments were carried out at room temperature.

\section{RESULTS AND DISCUSSION}


Small Angle X-ray Scattering (SAXS) patterns (1-D and 2-D) of lyotropic templates of phytantriol at $55{ }^{\circ} \mathrm{C}$, and phytantriol containing $4.1 \%(\mathrm{w} / \mathrm{w})$ 9-cis-tricosene at $25{ }^{\circ} \mathrm{C}$, both in excess hexachloroplatinic acid (HCPA) solution, are shown in Figure 1. The 1-D SAXS patterns each show three Bragg peaks with relative positions for $1 / \mathrm{d}$ in the ratios $\sqrt{1}: \sqrt{ } 3: \sqrt{ } 4$ which can be indexed as $(\mathrm{hkl})=(100),(111),(200)$ consistent with the first three reflections of an $H_{I I}$ phase. The 2-D SAXS patterns are comprised of three sharp Debye-Scherrer rings which suggest the presence of well-defined polydomain hexagonal liquid crystalline structures.

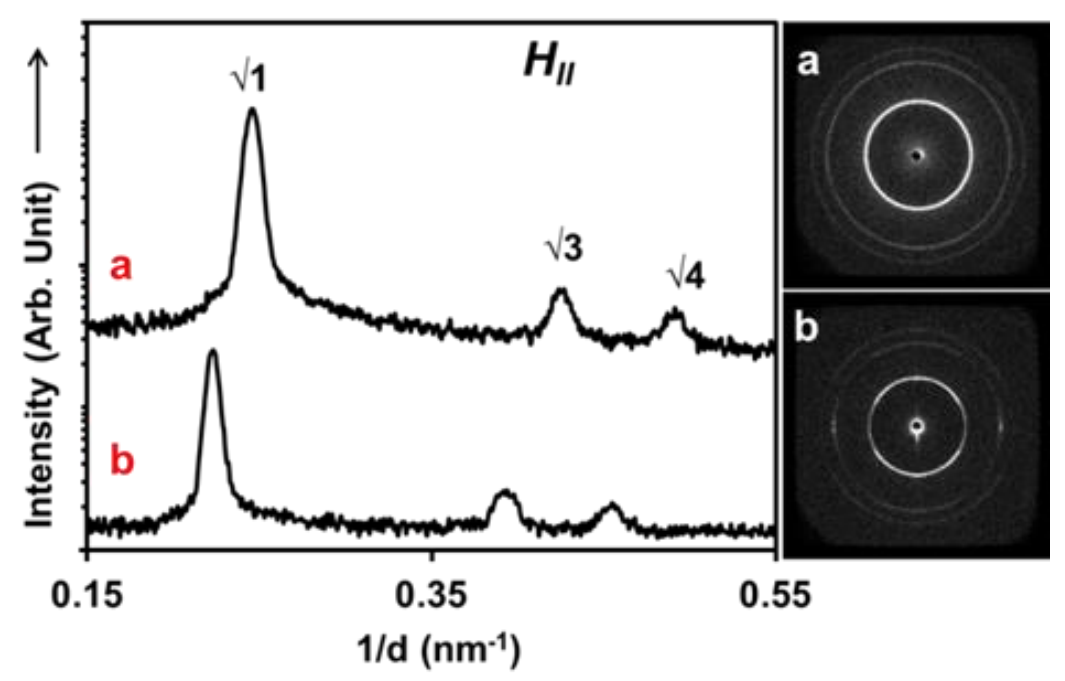

Figure 1. 1-D (left) and 2-D (right) SAXS patterns for a) phytantriol in excess HCPA solution at $55^{\circ} \mathrm{C}$ and b) phytantriol containing $4.1 \%(\mathrm{w} / \mathrm{w})$ 9-cis-tricosene in excess HCPA solution at 25 ${ }^{\circ} \mathrm{C}$. SAXS patterns in excess water are shown in Supplementary Information (Figure S1).

The lattice parameter, a, represents the centre-to-centre distance of adjacent cylinders, as shown in Figure 2. For the inverse hexagonal phase of phytantriol at $55^{\circ} \mathrm{C}$ in excess water and HCPA solution, lattice parameter values are $4.62 \pm 0.15$ and $4.73 \pm 0.15 \mathrm{~nm}$ respectively, indicating that the presence of hexachloroplatinate ions does not significantly interact with the lipid headgroups or affect mesophase structure. A similar observation was made previously with the inverse cubic phase $^{16}$. Lattice parameters for the inverse hexagonal phase of phytantriol with $4.1 \%$ 9-cis- 
tricosene at $25{ }^{\circ} \mathrm{C}$ in excess water and HCPA solution are $5.16 \pm 0.02$ and $5.24 \pm 0.02 \mathrm{~nm}$ respectively. The slightly higher lattice parameter at $25^{\circ} \mathrm{C}$ compared with $55^{\circ} \mathrm{C}$ is likely due to a decrease in spontaneous curvature ${ }^{27}$ due to a reduction in hydrocarbon splay, thus facilitating a swelling in the lattice parameter.

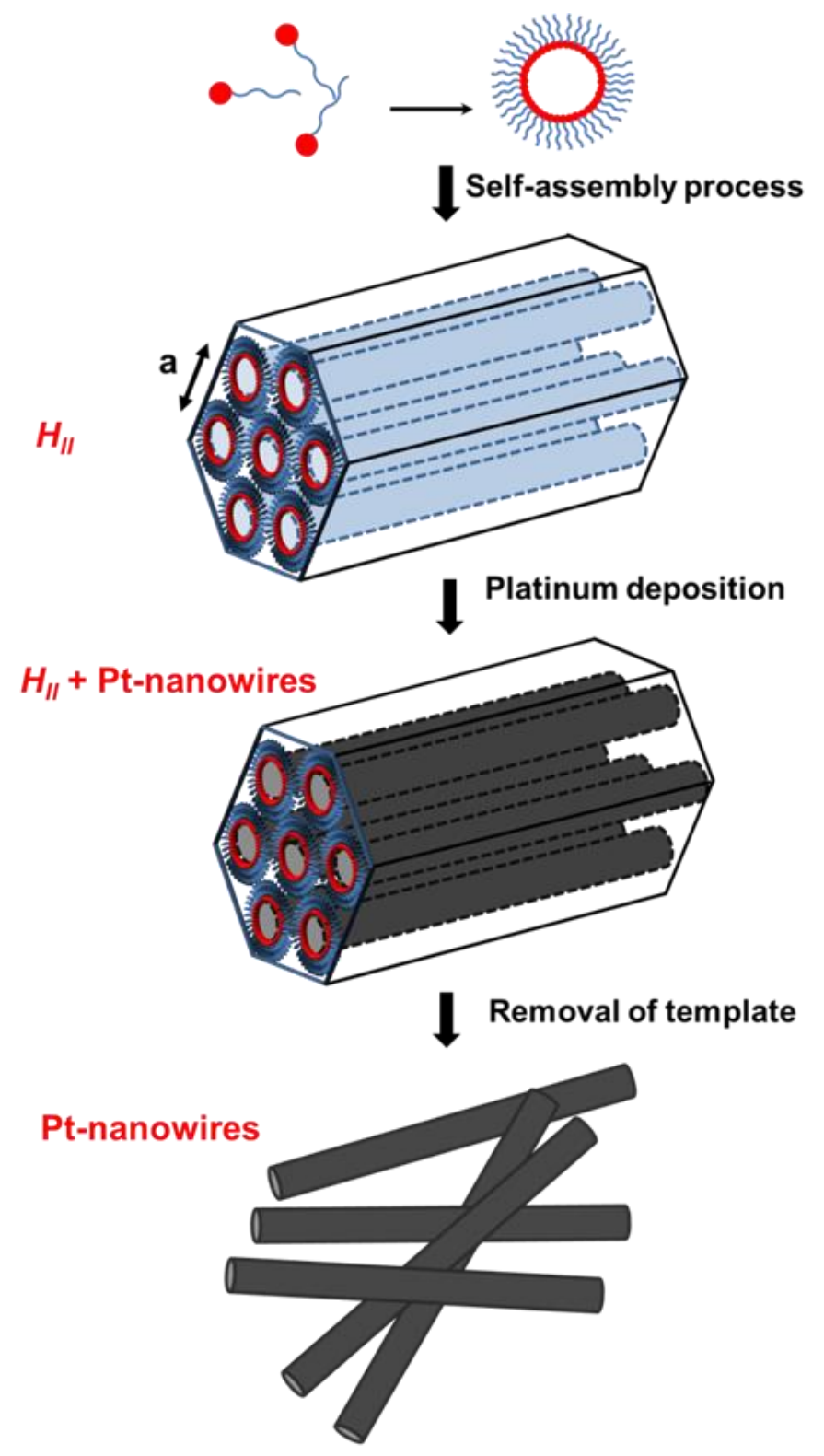

Figure 2. Schematic representation of fabrication of Pt-nanowires from $H_{\text {II }}$ phase of phytantriol, where 'a' represents the lattice parameter. Representative SAXS patterns for $H_{I I}$ phase, $H_{I I} / \mathrm{Pt}$ composite, and Pt-nanowires are shown in Supplementary Information Figure S2. 
The soft templated metal reduction route by galvanic displacement was first employed by Attard et $a .^{28}$ in 1997 to produce nanostructured platinum containing regular cylindrical mesopores. In the study reported here, the approach is employed for the first time to produce ultrathin Ptnanowires. Deposition of the platinum metal from HCPA dissolved in the aqueous channels of the $H_{I I}$ phase was achieved by galvanic displacement through contact with a stainless steel plate. Dissolution of the iron within the steel provides a source of electrons which reduces the HCPA to solid platinum constrained to grow within the cylindrical aqueous channel (Figure 2). As platinum reduction only takes place at a metal surface (initially the stainless steel, then at the growing end of the nanowire), this favours the growth of more uniform nanowires that grow longer and longer as deposition continues. In contrast, in reactions where platinum reduction is carried out by a reducing agent in the aqueous phase, multiple nucleation processes occur, producing shorter, more disordered nanowires ${ }^{29}$. The aqueous channels themselves are highly uniform, as shown by the sharp Bragg peaks in the diffraction patterns of the template (Figure 1). In order to investigate the possibility that the reduction of hexachloroplatinate ions and the growth of nanowires somehow affect the template, SAXS patterns for the $H_{I I}$ phase of phytantriol/ HCPA with incorporated Ptnanowires were taken at $55^{\circ} \mathrm{C}$ during the deposition process. No difference was observed in the SAXS patterns taken for the $H_{I I}$ phase of phytantriol/ HCPA before and after deposition (Supporting Information Figure S2). On removal of the template by dissolution of phytantriol in ethanol, the nanowires were no longer held in a hexagonal array, so no Bragg peaks were seen, as shown in Supporting Information Figure S2.

The morphology of the Pt-nanowires after template removal was characterised by low resolution and high resolution TEM. The micrographs of the well-defined individual Pt-nanowires fabricated in $H_{I I}$ phases at $55^{\circ} \mathrm{C}$, and at $25^{\circ} \mathrm{C}$ in the presence of $4.1 \%$ 9-cis-tricosene, are shown in Figure 
3. After removal of the template, the unsupported nanowires were observed to be held together to form bundles or entangled to form a mesh like structures as shown in Figure 3. The Pt-nanowires seem to be quite flexible as bending of the nanowires is quite visible from the TEM micrographs.

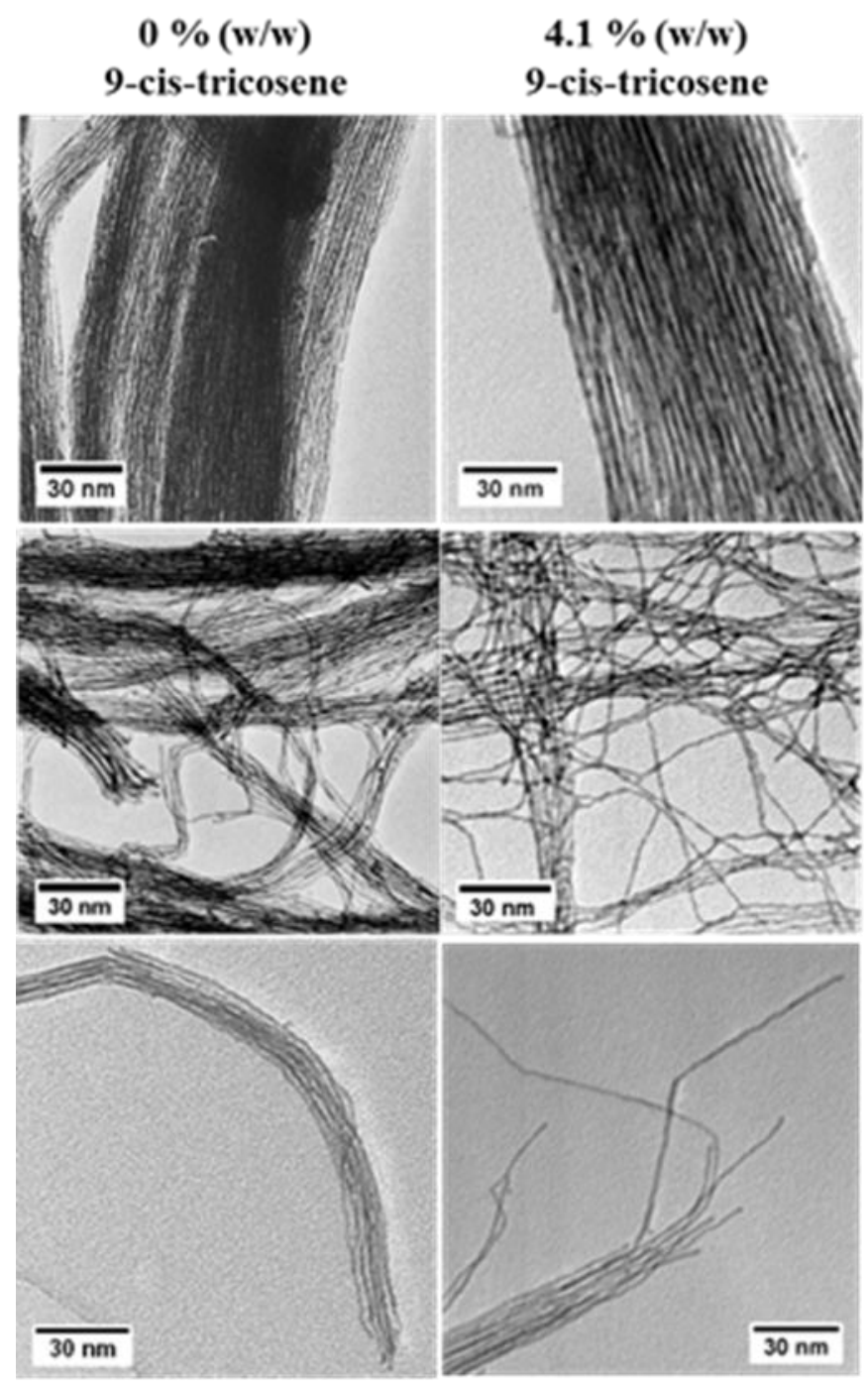

Figure 3. TEM micrographs of Pt-nanowires fabricated from the $H_{I I}$ phase of phytantriol in the presence of $0 \%$ (left) and $4.1 \%$ (right) (w/w) tricosene at 55 and $25{ }^{\circ} \mathrm{C}$ respectively. The fabrication time was 6 hours.

The TEM micrographs show that the nanowires exhibit a smooth and continuous surface morphology. Pt-nanowires were found to be at least hundreds of nanometres in length. Average 
diameters of the Pt-nanowires fabricated at different temperatures by the chemical reduction of HCPA in the aqueous mesochannels of the $H_{I I}$ phase of phytantriol containing 0 and $4.1 \%(\mathrm{w} / \mathrm{w})$ tricosene were estimated to be $2.5 \pm 0.4$ and $1.7 \pm 0.1 \mathrm{~nm}$ respectively. (These values were obtained from individual measured values of: $2.2,2.5,2.7,2.8,2.9,2.1$ and $2.4 \mathrm{~nm}$ for $0 \%$ tricosene; and $1.7,1.7,1.6,1.6,1.7$ and $1.8 \mathrm{~nm}$ for $4.1 \%(\mathrm{w} / \mathrm{w})$ tricosene. $)$

The dimensions of the aqueous channels in the $H_{I I}$ phase of the binary phytantriol/ HCPA solution system can be estimated using the following equation:

$$
r_{w}=\sqrt{\frac{\sqrt{3} a^{2} \phi_{w}}{2 \pi}}
$$

where $r_{w}$ is the radius of the aqueous channel, $a$ is the lattice parameter of the $H_{I I}$ phase, and $\phi_{w}$ is the aqueous water volume fraction. This equation is adapted from the corresponding formula for the radius of cylindrical surfactant micelles in the type I hexagonal lyotropic phase ${ }^{17}$. Detailed studies by Barauskas et al. on the phytantriol/ water system ${ }^{24}$ suggested that at $55{ }^{\circ} \mathrm{C}$ in excess water, the $H_{I I}$ phase is approximately $26 \% \mathrm{w} / \mathrm{w} \mathrm{H}_{2} \mathrm{O}$, and so $\phi_{w}=0.247$ (phytantriol density is $\left.0.932 \mathrm{~g} / \mathrm{cm}^{3}\right)$. Using a lattice parameter value of $a=4.7 \mathrm{~nm}$ gives an aqueous channel radius of $r_{w}=1.2 \mathrm{~nm}$, and therefore a diameter of $2.4 \mathrm{~nm}$, in good agreement with the size of the nanowires grown within it $(2.5 \pm 0.4 \mathrm{~nm}$ ). (For the room temperature system containing tricosene, we would expect the nanowires to be slightly bigger rather than smaller following the relative dimensions of the lipid template, as discussed above, but in this case no data are available for $\phi_{w}$ in excess water, so $r_{w}$ is not known.) As the Pt-nanowires were fabricated through a lyotropic liquid crystalline lipid template, the template could simply be removed by ethanol/acetone and water. Under these mild conditions, we avoid the structural degradation observed elsewhere by groups using hard templates for the synthesis of Pt-nanowires ${ }^{7}$. The agreement of platinum nanostructure dimensions with those of the lyotropic template is consistent with observations in normal topology hexagonal 
and cubic $^{17}$ and inverse cubic ${ }^{16}$ liquid crystal templating through electrodeposition, and suggests a rational approach for tuneable metal nanostructures by control of the template.

In order to demonstrate that the nanowires are indeed platinum, and to examine their individual structure, high resolution (HR) TEM studies were carried out. HR TEM micrographs of Ptnanowires fabricated at $55^{\circ} \mathrm{C}$ from the $H_{I I}$ phase of pure phytantriol are shown in Figure 4 .
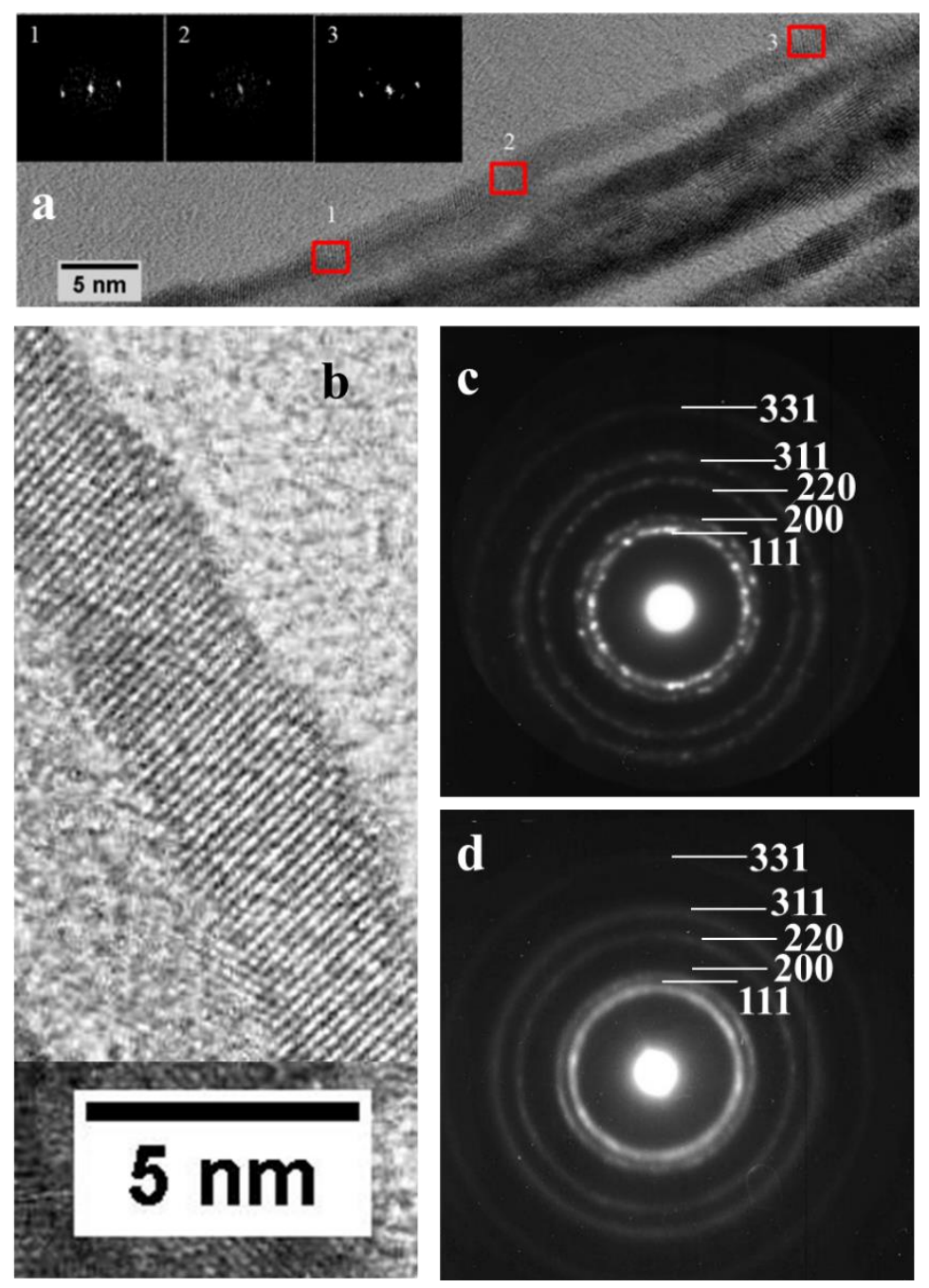

Figure 4. (a,b) HR TEM micrographs of Pt-nanowires synthesised at $55{ }^{\circ} \mathrm{C}$ in the absence of tricosene at different magnifications. Insets in (a) show three selected area FFT images $(1,2$, and 3 ), taken from the red boxed areas (1,2, and 3 respectively) of a single nanowire. (c,d) Selected 
area electron diffraction patterns taken from $230 \mathrm{~nm}$ regions of nanowire samples fabricated chemically in the presence of $0 \%$ (c) and $4.1 \%(w / w)$ tricosene (d) at 55 and $25{ }^{\circ} \mathrm{C}$ respectively.

Each Pt-nanowire showed lattice fringes which run parallel throughout the length of the nanowires, at least within the range of TEM micrograph $(50 \mathrm{~nm})$, revealing the crystalline nature of the nanowires. These crystals show different orientation in different nanowires. Therefore the selected area electron diffraction pattern (Figure 4) taken from a $230 \mathrm{~nm}$ region of parallel nanowires showed diffuse rings with $d$-spacings of $0.226,0.198,0.141,0.117$ and $0.0191 \mathrm{~nm}$, which can be indexed as $(h k l)=(111),(200),(220),(311)$, and (331) reflections of the fcc $\mathrm{Pt}^{6}$ respectively. In some cases, the crystallinity was well preserved in the neighbouring nanowires, showing that the Pt-nanowires were grown from a single metal crystal domain.

The nanowires were characterised electrochemically, to further confirm that they were made of platinum, and to measure the electrochemically accessible surface area of a sample. The characterization was carried out by cyclic voltammetry in dilute $\mathrm{H}_{2} \mathrm{SO}_{4}$ solution. For this purpose, the Pt-nanowires were rubbed onto a bare gold wire of diameter $0.2 \mathrm{~mm}$ to a length of approximately $2 \mathrm{~mm}$, and therefore with exposed electrode area $0.0129 \mathrm{~cm}^{2}$. The cyclic voltammograms (CVs) of the two nanowire samples produced at $55^{\circ} \mathrm{C}$ and at $25^{\circ} \mathrm{C}$ in the presence of tricosene are shown in Figure 5, together with the CV of the gold electrode with no Ptnanowires. 

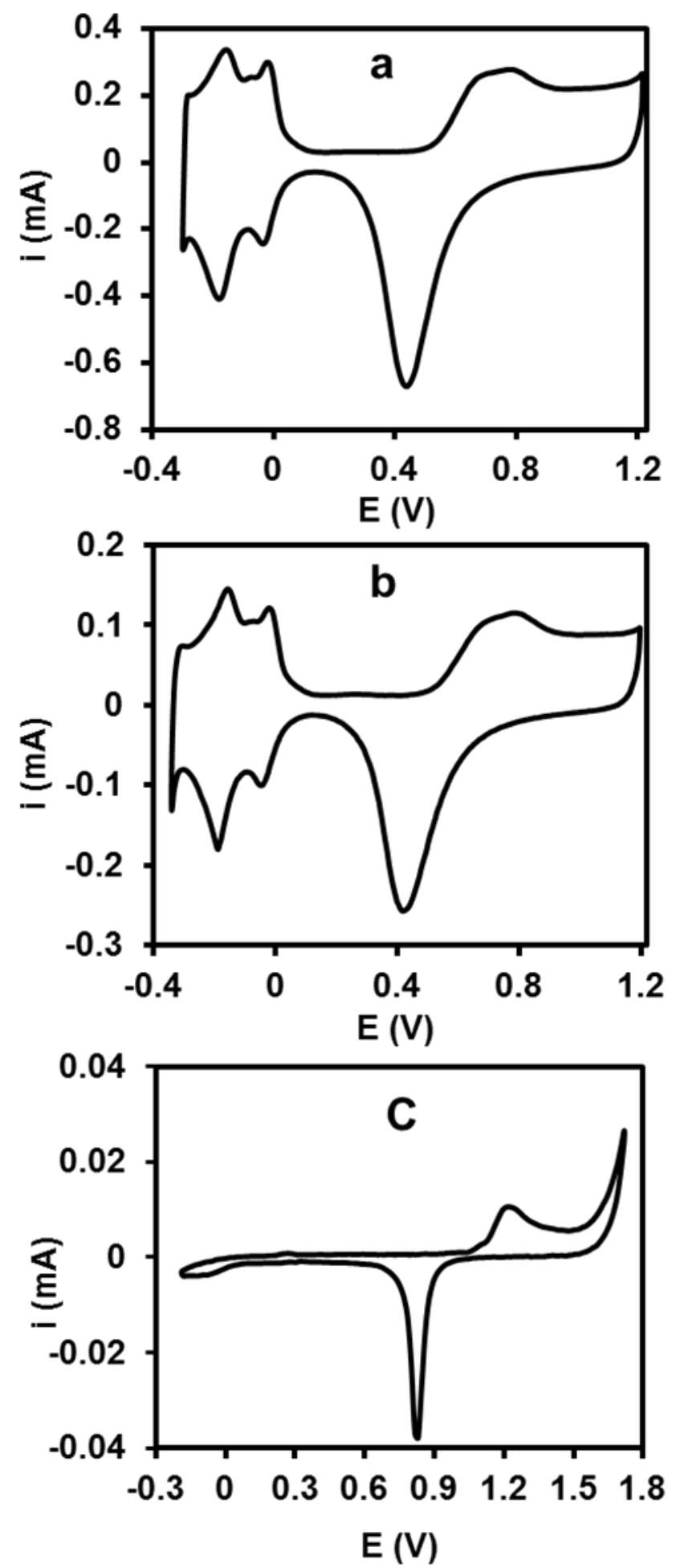

Figure 5. Cyclic voltammograms in $0.5 \mathrm{M}$ aqueous $\mathrm{H}_{2} \mathrm{SO}_{4}$ at $0.1 \mathrm{~V} \mathrm{~s}^{-1}$ between the limits +1.2 and $-0.3 \mathrm{~V}$ vs. $\mathrm{Ag} / \mathrm{AgCl}$ for Pt-nanowire rubbed gold wire electrodes. The Pt-nanowires were fabricated chemically in the presence of (a) 0 and (b) $4.1 \%(\mathrm{w} / \mathrm{w})$ tricosene at 55 and $25{ }^{\circ} \mathrm{C}$ 
respectively. A typical cyclic voltammogram of bare gold wire electrode before rubbing of Ptnanowires is shown as (c).

The presence of the nanowires produces a dramatic increase in current; the features at positive potentials are associated with a platinum oxide layer, while the multiple peaks at slightly negative potentials show the formation and removal of a hydrogen monolayer; the multiple peaks are associated with different Pt crystalline facets ${ }^{31}$ and the total peak area is proportional to the electrochemically active surface area of platinum, which can be estimated following calculations outlined in more detail elsewhere ${ }^{32}$. The CVs in Figure 5 give platinum surface areas of 3.5714 $\mathrm{cm}^{2}$ and $1.522 \mathrm{~cm}^{2}$ which means that rubbing the original $0.0129 \mathrm{~cm}^{2}$ gold electrode in Ptnanowires produced an increase by a factor of 277 and 118 in surface area from nanowires synthesised respectively at $55^{\circ} \mathrm{C}$, and $25{ }^{\circ} \mathrm{C}$ with tricosene. This suggests $3 \mathrm{D}$ electrochemically accessible networks of wires, rather than a tightly packed arrangement where only the top layer is accessible to the external solution. Differences in surface area between the two samples likely reflect variations in the amount of Pt-nanowire powder adhering to the gold electrode surface on rubbing. The specific surface area can be estimated geometrically from the nanowire dimensions.

Given the high aspect ratios of the nanowires, the contribution of the nanowire ends to surface area can be ignored. Therefore, for a nanowire radius $r$, for a section of length $\mathrm{L}$, the surface area is $2 \pi \mathrm{r} \mathrm{L}$, and the mass is $\pi \mathrm{r}^{2} \mathrm{~L} \rho$, where $\mathrm{r}$ is the radius, and $\rho$, the density of platinum, is $21.45 \times$ $10^{6} \mathrm{~g} / \mathrm{m}^{3}$. For nanowires of radius $\mathrm{r}=1.25 \times 10^{-9} \mathrm{~m}$ and $\mathrm{r}=0.85 \times 10^{-9} \mathrm{~m}$ (from TEM data), the specific surface area estimates are 75 and $110 \mathrm{~m}^{2} / \mathrm{g}$ respectively. For comparison, typical specific area values for platinum nanoparticles on carbon are around $60-70 \mathrm{~m}^{2} / \mathrm{g}^{33-34}$ while values of 71.34 $\mathrm{m}^{2} / \mathrm{g}$ and $90 \mathrm{~m}^{2} / \mathrm{g}$ were reported for platinum nanowires with comparable dimensions produced 
using the insulin fibril templating and solvothermal routes described previously ${ }^{5,12}$ using much less synthetically attractive routes than that described here.

\section{SUPPORTING INFORMATION}

SAXS patterns for phytantriol in excess $\mathrm{H}_{2} \mathrm{O}$ at $55^{\circ} \mathrm{C}$ and phytantriol containing $4.1 \%$ (w/w) 9cis-tricosene in excess $\mathrm{H}_{2} \mathrm{O}$ at $25^{\circ} \mathrm{C}$ (Figure S1), SAXS patterns for $H_{I I}$ phase, $H_{I I} / \mathrm{Pt}$ composited), and Pt-nanowires (Figure S2). Selected area electron diffraction patterns from a $230 \mathrm{~nm}$ region of Pt-nanowires fabricated chemically in the presence of 0 and $4.1 \%(\mathrm{w} / \mathrm{w})$ tricosene at 55 and $25{ }^{\circ} \mathrm{C}$ respectively and High Resolution TEM images (Figure S3).

\section{AUTHOR INFORMATION}

\section{Corresponding Authors}

Adam M. Squires

Department of Chemistry, University of Bath, Bath BA2 7AY, UK

E-mail: a.squires@bath.ac.uk

Joanne M. Elliott

Department of Chemistry, University of Reading, Whiteknights, Reading, RG6 6AD

E-mail: j.m.elliott@,reading.ac.uk

\section{Author Contributions}

The manuscript was written through contributions of all authors. All authors have given approval to the final version of the manuscript.

\section{Funding Sources}


SA was funded by a scholarship from the University of Engineering and Technology, Lahore

Pakistan.

\section{ACKNOWLEDGMENT}

(Word Style "TD_Acknowledgments"). SAXS and electron microscopy experiments were carried out at the University of Reading's Chemical Analysis Facility and Centre for Advanced Microscopy respectively.

\section{ABBREVIATIONS}

$H_{I I}$, Inverse hexagonal lyotropic liquid crystal phase; SAXS, Small Angle X-Ray Scattering; HCPA, Hexachloroplatinic acid; 1D, One dimensional; 2D, two dimensional; 3D, Three dimensional; HR TEM, High resolution Transmission electron microscope; FFT, Fast Fourier transform; CV, cyclic voltammetry.

\section{REFERENCES}

1. Antolini, E.; Perez, J., The renaissance of unsupported nanostructured catalysts for lowtemperature fuel cells: from the size to the shape of metal nanostructures. J. Mater. Sci. 2011, 46 (13), 4435-4457.

2. $\quad$ Li, L.-L.; Chang, C.-W.; Wu, H.-H.; Shiu, J.-W.; Wu, P.-T.; Diau, E. W.-G., Morphological control of platinum nanostructures for highly efficient dye-sensitized solar cells. J. Mater. Chem. 2012, 22 (13), 6267-6273.

3. Taurino, I.; Sanzó, G.; Mazzei, F.; Favero, G.; De Micheli, G.; Carrara, S., Fast synthesis of platinum nanopetals and nanospheres for highly-sensitive non-enzymatic detection of glucose and selective sensing of ions. Scientific Reports 2015, 5, 15277.

4. Cheong, S.; Watt, J. D.; Tilley, R. D., Shape control of platinum and palladium nanoparticles for catalysis. Nanoscale 2010, 2 (10), 2045-2053.

5. Zhang, L.; Li, N.; Gao, F.; Hou, L.; Xu, Z., Insulin Amyloid Fibrils: An Excellent Platform for Controlled Synthesis of Ultrathin Superlong Platinum Nanowires with High Electrocatalytic Activity. J. Am. Chem. Soc. 2012, 134 (28), 11326-11329.

6. Koenigsmann, C.; Zhou, W.-p.; Adzic, R. R.; Sutter, E.; Wong, S. S., Size-Dependent Enhancement of Electrocatalytic Performance in Relatively Defect-Free, Processed Ultrathin Platinum Nanowires. Nano Lett. 2010, 10 (8), 2806-2811.

7. Suzuki, T.; Miyata, H.; Noma, T.; Kuroda, K., Platinum Thin Film Consisting of WellAligned Nanowires and Its Optical Behavior. J. Phys. Chem. C 2008, 112 (6), 1831-1836. 
8. Teng, X.; Han, W.-Q.; Ku, W.; Hücker, M., Synthesis of Ultrathin Palladium and Platinum Nanowires and a Study of Their Magnetic Properties. Angew. Chem. 2008, 120 (11), 2085-2088.

9. Tong, D. H.; Tran, P. D.; Pham, X. T. T.; Pham, V. B.; Le, T. T. T.; Dang, M. C.; Rijn, C. J. M. V., The nanofabrication of Pt nanowire arrays at the wafer-scale and its application in glucose detection. Adv. Nat. Sci.: Nanosci. Nanotechnol. 2010, 1 (1), 015011.

10. Kundu, S.; Huitink, D.; Liang, H., Formation and Catalytic Application of Electrically Conductive Pt Nanowires. J. Phys. Chem. C 2010, 114 (17), 7700-7709.

11. Azulai, D.; Cohen, E.; Markovich, G., Seed Concentration Control of Metal Nanowire Diameter. Nano Lett. 2012, 12 (11), 5552-5558.

12. Xia, B. Y.; Wu, H. B.; Yan, Y.; Lou, X. W.; Wang, X., Ultrathin and ultralong singlecrystal platinum nanowire assemblies with highly stable electrocatalytic activity. J. Am. Chem. Soc. 2013, 135 (25), 9480-9485.

13. Wang, D.; Luo, H.; Kou, R.; Gil, M. P.; Xiao, S.; Golub, V. O.; Yang, Z.; Brinker, C. J.; Lu, Y., A General Route to Macroscopic Hierarchical 3D Nanowire Networks. Angew. Chem. 2004, 116 (45), 6295-6299.

14. Liang, H.-W.; Cao, X.; Zhou, F.; Cui, C.-H.; Zhang, W.-J.; Yu, S.-H., A Free-Standing PtNanowire Membrane as a Highly Stable Electrocatalyst for the Oxygen Reduction Reaction. $A d v$. Mater. 2011, 23 (12), 1467-1471.

15. Volosskiy, B.; Niwa, K.; Chen, Y.; Zhao, Z.; Weiss, N. O.; Zhong, X.; Ding, M.; Lee, C.; Huang, Y.; Duan, X., Metal-Organic Framework Templated Synthesis of Ultrathin, Well-Aligned Metallic Nanowires. ACS Nano 2015, 9 (3), 3044-3049.

16. Akbar, S.; Elliott, J. M.; Rittman, M.; Squires, A. M., Facile Production of Ordered 3D Platinum Nanowire Networks with "Single Diamond" Bicontinuous Cubic Morphology. Adv. Mater. 2013, 25 (8), 1160-1164.

17. Asghar, K. A.; Rowlands, D. A.; Elliott, J. M.; Squires, A. M., Predicting Sizes of Hexagonal and Gyroid Metal Nanostructures from Liquid Crystal Templating. ACS Nano 2015, 9 (11), 10970-10978.

18. Attard, G. S.; Leclerc, S. A. A.; Maniguet, S.; Russell, A. E.; Nandhakumar, I.; Bartlett, P. N., Mesoporous Pt/Ru Alloy from the Hexagonal Lyotropic Liquid Crystalline Phase of a Nonionic Surfactant. Chem. Mater. 2001, 13 (5), 1444-1446.

19. Song, Y.; Garcia, R. M.; Dorin, R. M.; Wang, H.; Qiu, Y.; Coker, E. N.; Steen, W. A.; Miller, J. E.; Shelnutt, J. A., Synthesis of Platinum Nanowire Networks Using a Soft Template. Nano Lett. 2007, 7 (12), 3650-3655.

20. Yaghmur, A.; de Campo, L.; Sagalowicz, L.; Leser, M. E.; Glatter, O., Emulsified Microemulsions and Oil-Containing Liquid Crystalline Phases. Langmuir 2004, 21 (2), 569-577.

21. Huang, L. M.; Wang, H. T.; Wang, Z. B.; Mitra, A.; Bozhilov, K. N.; Yan, Y. S., Nanowire Arrays Electrodeposited from Liquid Crystalline Phases. Adv. Mater. 2002, 14 (1), 61-64.

22. Huang, L.; Wang, H.; Wang, Z.; Mitra, A.; Zhao, D.; Yan, Y., Cuprite Nanowires by Electrodeposition from Lyotropic Reverse Hexagonal Liquid Crystalline Phase. Chem. Mater. 2002, 14 (2), 876-880.

23. Jiang, X.; Xie, Y.; Lu, J.; Zhu, L.; He, W.; Qian, Y., Simultaneous In Situ Formation of ZnS Nanowires in a Liquid Crystal Template by $\gamma$-Irradiation. Chem. Mater. 2001, 13 (4), 12131218.

24. Barauskas, J.; Landh, T., Phase Behavior of the Phytantriol/Water System. Langmuir 2003, 19 (23), 9562-9565. 
25. Phan, S.; Fong, W.-K.; Kirby, N.; Hanley, T.; Boyd, B. J., Evaluating the link between self-assembled mesophase structure and drug release. Int. J. Pharm. 2011, 421 (1), 176-182.

26. Huang, T. C.; Toraya, H.; Blanton, T. N.; Wu, Y., X-ray powder diffraction analysis of silver behenate, a possible low-angle diffraction standard. J. Appl. Crystallogr. 1993, 26 (2), 180184.

27. Shearman, G. C.; Ces, O.; Templer, R. H.; Seddon, J. M., Inverse lyotropic phases of lipids and membrane curvature. J. Phys.: Condens. Matter 2006, 18 (28), S1 105.

28. Attard, G. S.; Corker, J. M.; Göltner, C. G.; Henke, S.; Templer, R. H., Liquid-Crystal Templates for Nanostructured Metals. Angew. Chem. Int. Ed. 1997, 36 (12), 1315-1317.

29. Yamauchi, Y.; Momma, T.; Fuziwara, M.; Nair, S. S.; Ohsuna, T.; Terasaki, O.; Osaka, T.; Kuroda, K., Unique Microstructure of Mesoporous Pt (HI-Pt) Prepared via Direct Physical Casting in Lyotropic Liquid Crystalline Media. Chem. Mater. 2005, 17 (25), 6342-6348.

30. Chemical Book.

http://www.chemicalbook.com/ProductChemicalPropertiesCB2141592_EN.htm, Access date June 13, 2016.

31. Bard, A. J.; Faulkner, L. R., Electrochemical methods : fundamentals and applications. Wiley: New York, 1980.

32. Trasatti, S.; Petrii, O. A., Real surface area measurements in electrochemistry. $J$. Electroanal. Chem. 1992, 327 (1-2), 353-376.

33. Zhou, Y.; Yang, G.; Pan, H.-B.; Zhu, C.; Fu, S.; Shi, Q.; Du, D.; Cheng, X.; Yang, J.; Wai, C. M.; Lin, Y., Ultrasonic-assisted synthesis of carbon nanotube supported bimetallic Pt-Ru nanoparticles for effective methanol oxidation. Journal of Materials Chemistry A 2015, 3 (16), 8459-8465.

34. $\quad$ Cheng, C.-F.; Hsueh, H.-Y.; Lai, C.-H.; Pan, C.-J.; Hwang, B.-J.; Hu, C.-C.; Ho, R.-M., Nanoporous gyroid platinum with high catalytic activity from block copolymer templates via electroless plating. NPG Asia Mater 2015, 7, e170. 
Table of Contents/ Abstract Graphic

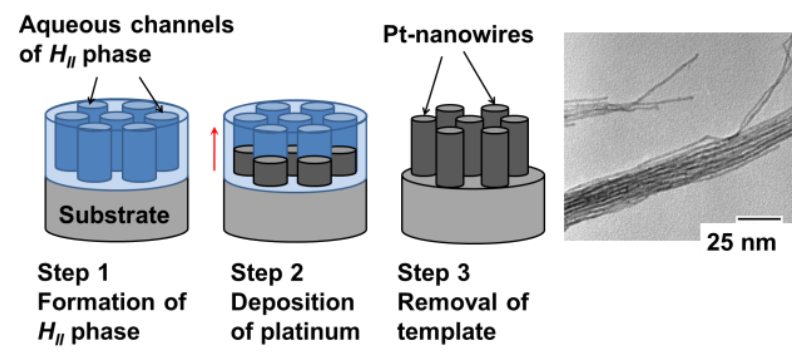

